\title{
Association of serum bilirubin levels with mortality in patients with diabetes initiating chronic hemodialysis: a competing risks analysis of a single-center cohort
}

Kiwako Toya, Tetsuya Babazono ${ }^{*}$, Hidekazu Murata, Ko Hanai and Yasuko Uchigata

\begin{abstract}
Background: Evidence remains scarce regarding the property of serum bilirubin levels as a prognostic factor for the incidence of mortality in dialysis patients. Here, we aimed to examine the association between serum bilirubin levels and mortality in patients with diabetes initiating hemodialysis.

Methods: This was a single-center, observational, historical cohort study of 395 Japanese patients with diabetes initiating chronic hemodialysis. There were 98 women and 297 men, and the mean ( \pm SD) age was $61 \pm 12$ years. Patients were dichotomized at the baseline median level of serum bilirubin $(0.3 \mathrm{mg} / \mathrm{dL})$. The endpoints were the incidence of all-cause and cause-specific mortality. Hazard ratios for these endpoints were estimated using the Fine-Gray subdistribution hazard model and cause-specific hazard model because of the presence of competing risks.

Results: During a median follow-up period of 3.9 years (range; 0.0-10.7 years), there were 83 cases of death, consisting of 39 and 44 cases from atherosclerotic cardiovascular diseases (ASCVDs) and non-ASCVDs, respectively. In the multivariate competing risks analysis, patients with lower bilirubin levels were associated with higher risk of ASCVD-related mortality (subdistribution hazard ratio 3.57, $p=0.015$ ) but not with non-ASCVD-related mortality $(0.83, p=0.665)$ than those with higher bilirubin levels. Analysis treating bilirubin levels as a continuous variable, as well as cause-specific models yielded the similar results.
\end{abstract}

Conclusions: Lower serum levels of bilirubin may be predictive of higher risk of ASCVD-related mortality in patients with diabetes initiating chronic hemodialysis. There was no relationship between levels of bilirubin and non-ASCVD-related mortality.

Keywords: Bilirubin, Diabetes mellitus, Hemodialysis, Mortality, Competing risks analysis

\section{Background}

Patients with diabetes undergoing dialysis still experience extremely high mortality $[1,2]$, the leading causes of which have been shown to be atherosclerotic cardiovascular diseases (ASCVDs) [3]. Several lines of evidence have suggested that increased oxidative stress is highly prevalent in patients receiving dialysis, contributing to the increased incidence of the adverse outcomes [4]. Two previous randomized, placebo-controlled trials of

\footnotetext{
* Correspondence: babazono.dmc@twmu.ac.jp

Diabetes Center, Tokyo Women's Medical University School of Medicine, 8-1 Kawada-cho, Shinjuku-ku, Tokyo 162-8666, Japan
}

patients undergoing hemodialysis have shown positive effects of antioxidant therapy on the development of ASCVDs [5, 6], despite a number of unsuccessful clinical trials using a range of interventions aimed to improve the prognosis in this population $[4,7]$.

Bilirubin possesses a potent antioxidant activity, effectively scavenging peroxyl radicals and suppressing oxidation of lipids and lipoproteins [8]. Clinical studies have shown that higher levels of serum bilirubin, even within normal range, are associated with reduced all-cause mortality as well as the incidence of ASCVD in patients without chronic kidney disease (CKD) $[9,10]$. We and 
others have suggested that higher serum levels of bilirubin possess protective effects on the incidence of microand macrovascular complications in diabetic patients [11-14]. In a cross-sectional study of Japanese dialysis patients with type 2 diabetes, lower serum bilirubin levels were significantly associated with higher presence of ASCVDs [15]; however, evidence remains scarce regarding property of serum bilirubin levels as a prognostic factor for the incidence of all-cause and ASCVDrelated mortality in dialysis patients with diabetes. Here, we aimed to examine the hypothesis that serum bilirubin levels are a predictor for mortality in diabetic patients initiating maintenance hemodialysis.

\section{Methods}

\section{Study design}

This was a single-center, longitudinal, observational study using a historical cohort of adult Japanese patients with diabetes initiating chronic hemodialysis. This study was designed in adherence to the Declaration of Helsinki and approved by the Ethics Committee of Tokyo Women's Medical University Hospital. Since this was an observational but not prospective intervention study, the Ethics Committee provided a waiver of informed consent. The present study used data from a clinical information system (electronic medical records).

\section{Participants}

From the hospital database, we initially collected data on 549 consecutive patients with type 1 and type 2 diabetes and specific types of diabetes due to other causes who newly initiated chronic hemodialysis at the Dialysis Unit, Department of Medicine, Diabetes Center, Tokyo Women's Medical University School of Medicine, Tokyo, Japan, during the period between January 2003 and March 2013. Types of diabetes were diagnosed according to the Japan Diabetes Society criteria [16]. In this study, we excluded patients if they had cholelithiasis $(n=7)$, liver cirrhosis $(n=7)$, chronic hepatitis B or C $(n=41)$, or malignant disease $(n=9)$ at baseline, as well as those with missing values for baseline profiles $(n=90)$. Overall, a total of 395 patients were included in the analysis.

\section{Measurements}

At the beginning of initial hemodialysis therapy, subjects underwent baseline anthropometric and physical examinations. Serum bilirubin levels were measured by an enzymatic method involving bilirubin oxidase using an automatic analyzer (Hitachi Labospect 008; Hitachi, Japan; normal range $0.2-1.2 \mathrm{mg} / \mathrm{dL}$ ). The lower measurement limitation of bilirubin was $0.1 \mathrm{mg} / \mathrm{dL}$; therefore, the levels less than $0.1 \mathrm{mg} / \mathrm{dL}$ in 12 patients were treated as $0.1 \mathrm{mg} / \mathrm{dL}$. High-density lipoprotein (HDL) cholesterol levels were determined using polyethylene glycol- pretreated enzymes, triglycerides using enzymatic methods, and low-density lipoprotein (LDL) cholesterol using enzymatic methods or Friedewald's equation (if levels of triglycerides $<400 \mathrm{mg} / \mathrm{dL}$ ). Serum creatinine concentrations were determined using an enzymatic method. HbA1c was measured utilizing a set of calibrators assigned by the Japan Diabetes Society (JDS) (normal range, 4.3-5.8\%); therefore, in the present study, National Glycohemoglobin Standardization Program (NGSP)-equivalent values were obtained using the following equation: HbA1c $(\%)=1.02 \times \mathrm{HbA1c}$ (JDS) (\%) + $0.25 \%$ [17]. GFR was estimated using the following equation, as proposed by the Japanese Society for Nephrology: estimated GFR (eGFR: $\left.\mathrm{mL} / \mathrm{min} / 1.73 \mathrm{~m}^{2}\right)=194 \times$ age (years) $)^{-0.287} \times$ serum creatinine level $(\mathrm{mg} / \mathrm{dL})^{-1.094} \times$ (0.739 if female) [18].

\section{Follow-up and endpoints}

After receiving initial several times of hemodialysis sessions at the dialysis unit of the hospital, all the patients were referred to and underwent chronic dialysis in outpatient hemodialysis clinics. Most of patients were also seen at the hospital thereafter for the purpose of glycemic control only.

The endpoints were the incidence of all-cause mortality and cause-specific mortality, the latter of which was classified into ASCVD-related and non-ASCVD-related mortality. The causes of ASCVD-related death were defined as follows: acute myocardial infarction, other ischemic heart disease including angina pectoris, heart failure, stroke such as cerebral hemorrhage and cerebral infarction, unhealed diabetic foot disease caused by peripheral arterial disease, and sudden death. Vital status of patients and cause of death in diseased patients were confirmed by direct reference to the hospital medical chart or inquiring to dialysis clinic. Patients were censored when they were lost to follow-up or at the administrative censoring date (April 30, 2014). Loss to follow-up was defined as the last visit 6 months or more before the administrative censoring date.

\section{Statistical analysis}

Data were expressed as percentage, arithmetic mean (standard deviation: SD), or median (range), as appropriate according to the data distribution. Student's $t$ test or Mann-Whitney $U$ test and Fisher's exact probability test were used to compare continuous and categorical data between two groups, respectively.

In consideration of the presence of competing risks, for example, death from causes other than ASCVDs in the analysis examining time to ASCVD-related death, cumulative incidence function (CIF) of mortality $[19,20]$ and the Fine and Gray subdistribution hazard model, as well as cause-specific hazard model [20,21], were used 
instead of the traditional Kaplan-Meier survival analysis and Cox proportional hazard model, respectively, as these latter methods may overestimate the risk of events by undermining the competing risks. In this study, kidney transplantation was also treated as a competing risk. In the multivariate models, the following covariates were incorporated: age, sex, duration of diabetes mellitus, history of ASCVDs, smoking status (current/ever versus never), date of hemodialysis initiation (January 2003 to December 2008 versus January 2009 to March 2013), body mass index, systolic blood pressure, diastolic blood pressure, HbA1c, logarithmically transformed triglycerides, HDL cholesterol, and LDL cholesterol.

$P$ values $<0.05$ were considered statistically significant. All statistical analyses were performed using SAS version 9.4 (SAS Institute, Cary, NC).

\section{Results}

Baseline characteristics

Of 395 patients studied, there were 98 women and 297 men, and the mean $( \pm \mathrm{SD})$ age was $61 \pm 12$ years (range 28-98 years). The vast majority (93.7 \%) had type 2 diabetes. Median known duration of diabetes was 18 years (range 1-60 years). Median serum bilirubin level was $0.3 \mathrm{mg} / \mathrm{dL}$ (range $0.1-0.8 \mathrm{mg} / \mathrm{dL}$ ), at which the subjects were dichotomized. Clinical characteristics and laboratory data in patients with higher and lower levels of serum bilirubin are presented in Table 1 . Those with lower bilirubin levels were likely to be younger, exhibiting higher frequencies of retinopathy and ASCVD, higher systolic blood pressure and LDL cholesterol levels, and lower hemoglobin levels than those with higher bilirubin levels.

Table 1 Baseline demographic and laboratory data in patients dichotomized at the median of serum bilirubin levels

\begin{tabular}{|c|c|c|c|}
\hline & Below the median $(n=191)$ & Above the median $(n=204)$ & $p$ value \\
\hline Age (years) & $59 \pm 13$ & $62 \pm 12$ & 0.012 \\
\hline Men (\%) & 73.8 & 76.5 & 0.562 \\
\hline Type 2 diabetes (\%) & 91.1 & 96.1 & 0.061 \\
\hline Duration of diabetes (years) & $18 \pm 9$ & $19 \pm 10$ & 0.617 \\
\hline Diabetic retinopathy (\%) & 92.7 & 77.5 & $<0.001$ \\
\hline Medication for diabetes (none/oral/insulin) & 25.1/6.3/68.6 & 25.5/17.2/57.3 & 0.002 \\
\hline BMI $\left(\mathrm{kg} / \mathrm{m}^{2}\right)$ & $24.7 \pm 4.2$ & $24.9 \pm 4.8$ & 0.648 \\
\hline Systolic blood pressure $(\mathrm{mmHg})$ & $157 \pm 24$ & $151 \pm 19$ & 0.007 \\
\hline Diastolic blood pressure (mmHg) & $78 \pm 13$ & $77 \pm 13$ & 0.764 \\
\hline Smoking (current or ever, \%) & 62.3 & 58.8 & 0.537 \\
\hline ASCVD (\%) & 57.1 & 44.1 & 0.012 \\
\hline \multicolumn{4}{|l|}{ Date of hemodialysis initiation } \\
\hline January 2003-December 2008 & 77.5 & 33.3 & \multirow[t]{2}{*}{$<0.001$} \\
\hline January 2009-March 2013 & 22.5 & 66.7 & \\
\hline \multicolumn{4}{|l|}{ Laboratory data } \\
\hline Hemoglobin (g/dL) & $8.8 \pm 1.2$ & $9.3 \pm 1.2$ & $<0.001$ \\
\hline HbA1c (NGSP) (\%) & $6.2 \pm 1.1$ & $6.3 \pm 1.0$ & 0.522 \\
\hline Triglycerides (mg/dL) & $125(38-1358)$ & $114(29-887)$ & 0.163 \\
\hline HDL cholesterol (mg/dL) & $42 \pm 15$ & $44 \pm 14$ & 0.202 \\
\hline LDL cholesterol (mg/dL) & $106 \pm 39$ & $96 \pm 36$ & 0.009 \\
\hline Albumin (g/dL) & $3.0 \pm 0.5$ & $3.2 \pm 0.4$ & $<0.001$ \\
\hline Creatinine (mg/dL) & $8.58 \pm 2.25$ & $8.26 \pm 1.89$ & 0.123 \\
\hline eGFR $\left(\mathrm{mL} / \mathrm{min} / 1.73 \mathrm{~m}^{2}\right)$ & $5.7 \pm 1.5$ & $6.0 \pm 2.5$ & 0.130 \\
\hline Total bilirubin (mg/dL) & $0.2(0.1-0.2)$ & $0.4(0.3-0.8)$ & $<0.001$ \\
\hline AST (U/L) & $17 \pm 10$ & $17 \pm 8$ & 0.977 \\
\hline $\mathrm{ALT}(\mathrm{U} / \mathrm{L})$ & $13 \pm 12$ & $12 \pm 9$ & 0.593 \\
\hline p-GT (U/L) & $18(5-235)$ & $17(7-416)$ & 0.828 \\
\hline
\end{tabular}

Data are expressed as percentage, mean $\pm \mathrm{SD}$, or median (range) $B M I$ body mass index, ASCVD atherosclerotic cardiovascular disease, eGFR estimated glomerular filtration rate, AST aspartate transaminase, $A L T$ alanine transaminase, $\gamma$-GT $\gamma$-glutamyl transferase 


\section{Association between bilirubin and mortality}

During a median follow-up period of 3.9 years (range 0.0-10.7 years), there were 83 deceased cases, in which 39 and 44 resulted from ASCVD and non-ASCVD, respectively. The causes of 44 non-ASCVD-related deaths were as follows: cancer $(n=8)$, infection $(n=14)$, disseminated intravascular coagulation $(n=1)$, refusal of receiving hemodialysis $(n=1)$, and unknown etiology $(n=20)$. Twenty patients underwent kidney transplantation, and 42 patients were lost to follow-up.

CIF of all-cause mortality tended to be higher in patients with lower bilirubin levels than those with higher bilirubin levels (Gray's test: $p=0.054$, Fig. 1a). Cumulative incidence of ASCVD-related mortality was significantly higher in those with lower bilirubin levels $(p=0.005$, Fig. 1b). No significant difference was observed in the CIF of non-ASCVD-related mortality $(p=0.807$, Fig. 1c).

As shown in Table 2, multivariate subdistribution hazard ratio for patients with lower versus higher bilirubin levels was $3.57(p=0.015)$ for ASCVD-related mortality with statistical significance and $1.56(p=0.162)$ for allcause mortality with marginal significance. Hazard ratio for non-ASCVD-related mortality was not statistically significant. Similar results were obtained in the analysis treating bilirubin levels as a continuous variable, indicating that every $0.1-\mathrm{mg}$ decrease in serum bilirubin level was associated with 2.3 times $(p=0.011)$ and 1.3 times $(p=0.121)$ higher risk of ASCVD-related mortality and all-cause mortality, respectively (Table 3 ). Results of cause-specific hazard models were almost identical to those of subdistribution models (Tables 2 and 3).

\section{Discussion}

In this single-center, observational, historical cohort study of Japanese patients with diabetes starting chronic hemodialysis, we found a significant association of lower versus higher serum bilirubin levels with 2.3 times higher risk of ASCVD-related mortality. Lower serum levels of bilirubin were also associated with higher risk of all-cause mortality; however, the hazard ratio was not statistically significant. These negative relationships were confirmed by treating bilirubin levels as a continuous variable. Furthermore, these relationships were independent of other variables that are well-known risk factors for the development of ASCVDs. In contrast, there was no relationship between levels of bilirubin and nonASCVD-related mortality.

A previous cohort study from Taiwan first identified lower bilirubin levels as a risk factor for incident ASCVD-related and all-cause mortality in dialysis patients with non-specific underlying kidney diseases [22]. Our study restricting to patients with diabetes yielded the consistent results; however, the previous study used conventional Kaplan-Meier survival analysis and Cox proportional hazard model for estimating the event-free survival and hazard ratio, despite the presence of competing risks including kidney transplantation and losses to follow-up. These classical survival analyses have been shown to be inappropriate in the presence of competing risks, yielding the potential for biases [22]. To overcome these limitations, we used competing risks analysis and confirmed that the inverse association of serum bilirubin and ASCVD-related mortality was robust.
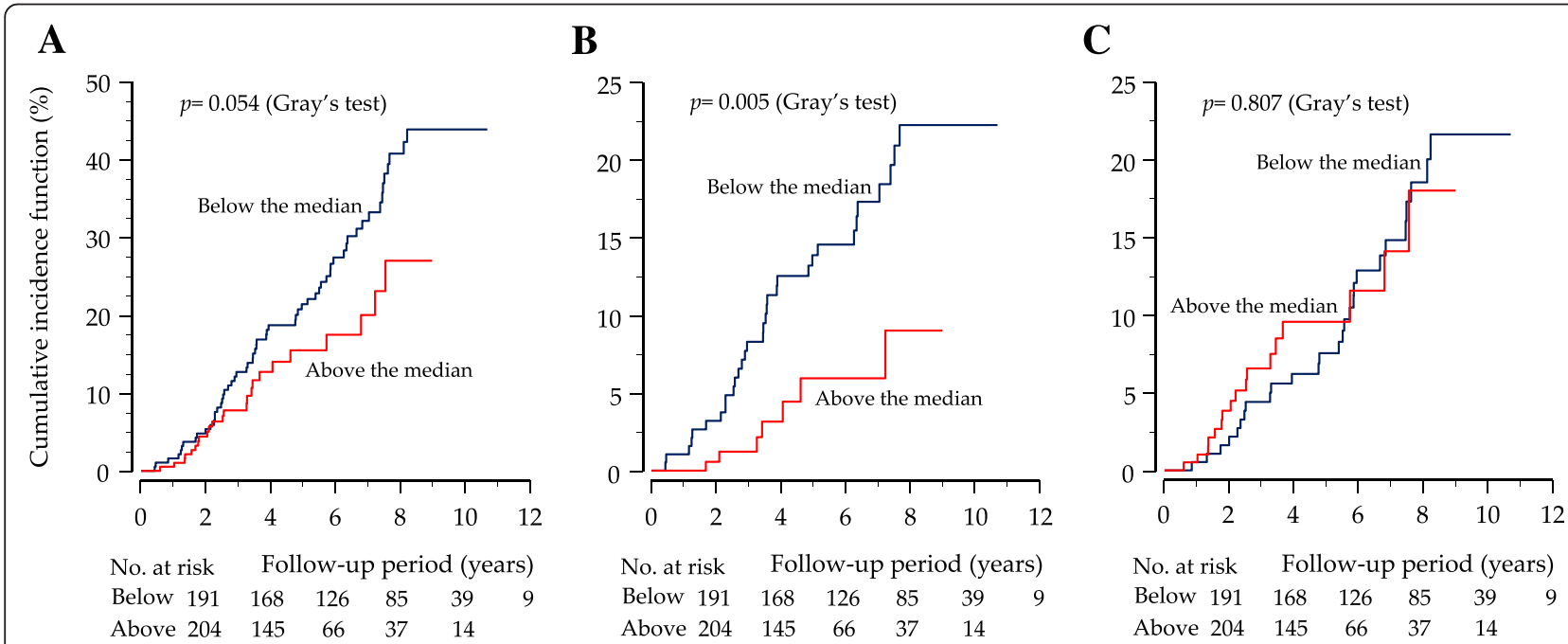

Fig. 1 a Cumulative incidence of the all-cause mortality between the lower and higher bilirubin groups estimated using the cumulative incidence function ( $p=0.054$, Gray's test). $\mathbf{b}$ Cumulative incidence of the atherosclerotic cardiovascular disease (ASCVD)-related mortality between the lower and higher bilirubin groups estimated using the cumulative incidence function ( $p=0.005$, Gray's test). c Cumulative incidence of the non-ASCVD-related mortality between the lower and higher bilirubin groups estimated using the cumulative incidence function ( $p=0.807$, Gray's test) 
Table 2 Hazard ratio for patients with lower versus higher bilirubin levels

\begin{tabular}{lllllllll}
\hline $\begin{array}{l}\text { Cause of } \\
\text { death }\end{array}$ & \multicolumn{3}{l}{ Subdistribution model } & & \multicolumn{3}{c}{ Cause-specific model } \\
\cline { 2 - 3 } \cline { 6 - 8 } & HR $(95 \% \mathrm{Cl})$ & $p$ value & & HR $(95 \% \mathrm{Cl})$ & $p$ value \\
\hline All causes & 1.56 & $(0.83-2.94)$ & 0.162 & & 1.45 & $(0.83-2.50)$ & 0.192 \\
ASCVD & 3.57 & $(1.28-10.00)$ & 0.015 & & 3.23 & $(1.30-8.33)$ & 0.013 \\
Non-ASCVD & 0.83 & $(0.37-1.89)$ & 0.665 & & 0.83 & $(0.40-1.72)$ & 0.609
\end{tabular}

Patients were dichotomized at the median $(0.3 \mathrm{mg} / \mathrm{dL})$ of bilirubin levels. Hazard ratios for mortality in patients with lower versus higher bilirubin levels were estimated using the Fine-Gray subdistribution hazard model and causespecific hazard model. The following variables were incorporated as independent variables: age, sex, duration of diabetes mellitus, history of ASCVD, smoking, date of hemodialysis initiation, body mass index, systolic blood pressure, diastolic blood pressure, $\mathrm{HbA1c}$, hemoglobin, serum albumin, logarithmically transformed triglycerides, high-density lipoprotein cholesterol, and low-density lipoprotein cholesterol

$H R$ hazard ratio, $\mathrm{Cl}$ confidence interval, $A S C V D$ atherosclerotic cardiovascular disease

Our results are inextricably linked to a number of studies showing beneficial effects of bilirubin on ASCVDs in patients with several conditions $[9,11,12,15]$; however, these observational studies including ours are unable to infer the causal relationship between levels of bilirubin and the outcomes. In this study, patients with lower levels of bilirubin were likely to manifest higher systolic blood pressure and LDL cholesterol levels at baseline; however, the effects of bilirubin were independent of these potential risk factors for ASCVDs. The most likely explanation may be that bilirubin possesses as a potent antioxidant, effectively scavenging peroxyl radicals and suppressing oxidation of lipids and lipoproteins [8]. In a large population-based cohort study from the UK, patients with Gilbert syndrome, a common disorder of unconjugated hyperbilirubinemia caused by a mutation in the gene promoter for bilirubin uridine glucuronyl transferase 1A1 (UGT1A1) [23], tended to have a much lower mortality rate than patients without evidence of the condition [24]. Total bilirubin levels and the bilirubin-associated UGT1A SNPs tended to be associated with all-cause mortality in patients with type

Table 3 Hazard ratio for of $0.1 \mathrm{mg} / \mathrm{dL}$ decrease in serum bilirubin levels

\begin{tabular}{|c|c|c|c|c|c|c|}
\hline \multirow{3}{*}{$\begin{array}{l}\text { Cause of } \\
\text { death } \\
\text { All causes }\end{array}$} & \multicolumn{3}{|c|}{ Subdistribution model } & \multicolumn{3}{|c|}{ Cause-specific model } \\
\hline & \multicolumn{2}{|c|}{ HR $(95 \% \mathrm{Cl})$} & \multirow{2}{*}{$\begin{array}{l}P \text { value } \\
0.121\end{array}$} & \multicolumn{2}{|c|}{$\overline{\mathrm{HR}}(95 \% \mathrm{Cl})$} & \multirow{2}{*}{$\frac{P \text { value }}{0.107}$} \\
\hline & 1.28 & $(0.93-1.75)$ & & 1.25 & $(0.95-1.61)$ & \\
\hline ASCVD & 2.27 & $(1.20-4.35)$ & 0.011 & 2.17 & $(1.35-3.57)$ & 0.002 \\
\hline Non-ASCVD & 0.88 & $(0.62-1.27)$ & 0.507 & 0.88 & $(0.63-1.23)$ & 0.451 \\
\hline
\end{tabular}

Hazard ratios for $0.1 \mathrm{mg} / \mathrm{dL}$ decrease in serum bilirubin levels for mortality was estimated using the Fine-Gray subdistribution hazard model and cause-specific model. The following variables were incorporated as independent variables: age, sex, duration of diabetes mellitus, history of ASCVD, smoking, date of hemodialysis initiation, body mass index, systolic blood pressure, diastolic blood pressure, $\mathrm{HbA1c}$, hemoglobin, serum albumin, logarithmically transformed triglycerides, high-density lipoprotein cholesterol, and low-density lipoprotein cholesterol $H R$ hazard ratio, $\mathrm{Cl}$ confidence interval, ASCVD atherosclerotic cardiovascular disease
2 diabetes without kidney disease [25]. Furthermore, the above Taiwanese cohort study of hemodialysis patients showed that UGT1A1 gene polymorphism had strong effects on serum bilirubin levels and also influenced the incidence of ASCVD and all-cause mortality [22]. These clinical studies strongly implicate lower levels of bilirubin in the pathogenesis of ACSCVDs through increased oxidant properties.

In this study, the association between bilirubinemia and non-ASCVD mortality, mainly from cancer and infection, was not observed. Cohort studies examining the association of bilirubin with incidence of and mortality from cancer have resulted in conflicting findings [26-28]. In our study, there were only eight cases with cancer-related mortality, limiting meaningful analysis to determine the cause-specific associations. Regarding infectious disease, bilirubin has also been known to have anti-inflammatory effects. The defensive role of bilirubin on infection was first shown almost 8 decades ago [29]; however; whether hypobilirubinemia is associated with higher incidence of infectious diseases, especially in patients with diabetes and end-stage renal disease (ESRD), is largely unknown. Future investigations including a large sample size are needed to determine the association in this population.

In agreement with a previous study [15], serum levels of bilirubin in the patients with ESRD were lower, raising possibilities that some uremic substrates interfere in the measurement and alter metabolism of serum bilirubin. To the best of our knowledge, we were unable to find these possibilities. Alternatively, another possibility is that hypobilirubinemia may be one of the causative factors in the development of ESRD in diabetic patients [11-14].

The present study has several limitations. First, we examined an ethnically homogenous population at a single urban university hospital in Japan; therefore, generalization of the present findings may be limited. Second, we were unable to completely exclude patients with hepatobiliary or hemolytic disease. Third, we did not evaluate time-dependent changes in serum bilirubin levels, lipid parameters, $\mathrm{HbA1c}$, blood pressure, BMI, or change in medications during follow-up. Fourth, we did not differentiate direct and indirect bilirubin from total serum bilirubin. We also did not evaluate any genetic factors which regulate bilirubin concentration. Fifth, 90 patients with missing values at baseline were excluded, 42 patients were lost to follow-up, and the causes of 20 cases among 44 non-ASCVD-related deaths were unknown etiology, which may add some bias in the analyses. Finally, this study was carried out using a relatively small cohort.

\section{Conclusions}

The present single-center observational cohort study may provide evidence for the clinical usefulness of 
serum bilirubin levels, easily measured in clinical practice, as the predictor for ASCVD-related mortality in patients with diabetes initiating hemodialysis. Meanwhile, bilirubin levels were not associated with the incidence of non-ASCVD-related mortality. The present findings should be confirmed in further studies including a larger sample size and a multicenter design. We believe that the present findings lead to the possibility of the intervention targeting bilirubin.

\section{Acknowledgements}

The authors thank Research Assistant Professor Kengo Nagashima, Department of Global Clinical Research, Graduate School of Medicine, Chiba University, for his professional advice on the statistical analysis.

\section{Funding}

None declared.

\section{Availability of data and materials}

The datasets analyzed during the present study are available from the corresponding author on reasonable request.

\section{Authors' contributions}

$\mathrm{KT}$ conceived the study, designed the protocol, contributed to the data collection and preparation, analyzed all data, wrote the manuscript, contributed to the interpretation of the results, and approved the final version. TB designed the protocol, analyzed all data, wrote the manuscript, contributed to the interpretation of the results, and approved the final version. HM collected the clinical data, contacted to dialysis units to confirm outcomes, and approved the final version. $\mathrm{KH}$ analyzed all data, wrote the manuscript, contributed to the interpretation of the results, and approved the final version. YU contributed to the interpretation of the results and approved the final version.

\section{Competing interests}

The authors declare that they have no competing interests.

\section{Consent for publication}

Not applicable.

\section{Ethics approval and consent to participate}

All procedures performed followed were in accordance with the ethical standards of the Ethics Committee of Tokyo Women's Medical University Hospital and the national research committee at which the studies were conducted (Approval No. 3932) and with the Helsinki declaration of 1964 and later versions. Since this was an observational, historical but not prospective intervention study, the Ethics Committee provided a waiver of informed consent.

\section{Received: 3 July 2016 Accepted: 20 August 2016}

\section{Published online: 28 October 2016}

\section{References}

1. Australia and New Zealand Dialysis and Renal Transplant Registry. http:// www.anzdata.org.au/anzdata/AnzdataReport/38thReport/c03_anzdata_ deaths_v2.0_20160112_web.pdf, Accessed 11 Feb 2016.

2. United States Renal Data System. 2015 USRDS annual data report: epidemiology of kidney disease in the United States. Bethesda: National Institutes of Health, National Institute of Diabetes and Digestive and Kidney Diseases; 2015

3. Villar E, Chang SH, McDonald SP. Incidences, treatments, outcomes, and sex effect on survival in patients with endstage renal disease by diabetes status in Australia and New Zealand (1991-2005). Diabetes Care. 2007;30:3070-6.

4. Coombes JS, Fassett RG. Antioxidant therapy in hemodialysis patients: a systematic review. Kidney Int. 2012;81:233-46.

5. Boaz M, Smetana S, Weinstein T, Matas Z, Gafter U, laina A, Knecht A, Weissgarten Y, Brunner D, Fainaru M, Green MS. Secondary prevention with antioxidants of cardiovascular disease in endstage renal disease (SPACE): randomised placebo-controlled trial. Lancet. 2000;356:1213-8.
6. Tepel M, van der Giet M, Statz M, Jankowski J, Zidek W. The antioxidant acetylcysteine reduces cardiovascular events in patients with end-stage renal failure: a randomized, controlled trial. Circulation. 2003;107:992-5.

7. Himmelfarb J, Ikizler TA, Ellis C, Wu P, Shintani A, Dalal S, Kaplan M, Chonchol M, Hakim RM. Provision of antioxidant therapy in hemodialysis (PATH): a randomized clinical trial. J Am Soc Nephrol. 2014;25:623-33.

8. Stocker R, Yamamoto Y, McDonagh AF, Glazer AN, Ames BN. Bilirubin is an antioxidant of possible physiological importance. Science. 1987; 235:1043-6.

9. Horsfall L, Nazareth I, Petersen I. Cardiovascular events as a function of serum bilirubin levels in a large, statin-treated cohort. Circulation. 2012;126:2556-64.

10. Ong KL, Allison MA, Cheung BMY, Wu BJ, Barter PJ, Rye KA. The relationship between total bilirubin levels and total mortality in older adults: the United States National Health and Nutrition Examination Survey (NHANES) 1999-2004. PLoS One. 2014;9:e94479.

11. Fukui M, Tanaka M, Shiraishi E, Harusato I, Hosoda H, Asano M, Hasegawa G, Nakamura N. Relationship between serum bilirubin and albuminuria in patients with type 2 diabetes. Kidney Int. 2008;74:1197-201.

12. Chan KH, O'Connell RL, Sullivan DR, Hoffmann LS, Rajamani K, Whiting M, Donoghoe MW, Vanhala M, Hamer A, Yu B, Stocker R, Ng MK, Keech AC. Plasma total bilirubin levels predict amputation events in type 2 diabetes mellitus: the Fenofibrate Intervention and Event Lowering in Diabetes (FIELD) study. Diabetologia. 2013;56:724-36.

13. Toya K, Babazono T, Hanai K, Uchigata Y. Association of serum bilirubin levels with development and progression of albuminuria, and decline in estimated glomerular filtration rate in patients with type 2 diabetes mellitus. J Diabetes Invest. 2014;5:228-35.

14. Sekioka $R$, Tanaka M, Nishimura T, Itoh H. Serum total bilirubin concentration is negatively associated with increasing severity of retinopathy in patients with type 2 diabetes mellitus. J Diabetes Complications. 2015;29:218-21.

15. Fukui M, Tanaka M, Yamazaki M, Hasegawa G, Nishimura M, Iwamoto N Ono T, Imai S, Nakamura N. Low serum bilirubin concentration in haemodialysis patients with type 2 diabetes. Diabet Med. 2011;28:96-9.

16. Kuzuya T, Nakagawa S, Satoh J, Kanazawa Y, Iwamoto Y, Kobayashi M, Nanjo K, Sasaki A, Seino Y, Ito C, Shima K, Nonaka K, Kadowaki T. Committee of the Japan Diabetes Society on the diagnostic criteria of diabetes mellitus. Report of the committee on the classification and diagnostic criteria of diabetes mellitus. Diabetes Res Clin Pract. 2002;55:65-85.

17. Kashiwagi A, Kasuga M, Araki E, Oka Y, Hanafusa T, Ito H, Tominaga M, Oikawa S, Noda M, Kawamura T, Sanke T, Namba M, Hashiramoto M, Sasahara T, Nishio Y, Kuwa K, Ueki K, Takei I, Umemoto M, Murakami M, Yamakado M, Yatomi Y, Ohashi H, Committee on the Standardization of Diabetes Mellitus-Related Laboratory Testing of Japan Diabetes Society. International clinical harmonization of glycated hemoglobin in Japan: from Japan Diabetes Society to National Glycohemoglobin Standardization Program values. J Diabetes Invest. 2012;3:39-40.

18. Matsuo S, Imai E, Horio M, Yasuda Y, Tomita K, Nitta K, Yamagata K, Tomino Y Y Yokoyama H, Hishida A. Collaborators developing the Japanese equation for estimated GFR. Revised equations for estimated GFR from serum creatinine in Japan. Am J Kidney Dis. 2009:53:982-92.

19. Kalbfleisch JD, Prentice RL. The statistical analysis of failure time data. 2nd ed. New York: John Wiley and Sons; 2002.

20. Austin PC, Lee DS, Fine JP. Introduction to the analysis of survival data in the presence of competing risks. Circulation. 2016;133:601-9.

21. Fine JP, Gray RJ. A proportional hazards model for the sub-distribution of a competing risk. J Am Stat Assoc. 1999;94:496-509.

22. Chen YH, Hung SC, Tarng DC. Serum bilirubin links UGT1A1*28 polymorphism and predicts long-term cardiovascular events and mortality in chronic hemodialysis patients. Clin J Am Soc Nephrol. 2011;6:567-74.

23. Bosma PJ, Chowdhury JR, Bakker C, Gantla S, de Boer A, Oostra BA, Lindhout D, Tytgat GNJ, Jansen PLM, Oude Elferink RP, Chowdhury NR. The genetic basis of the reduced expression of bilirubin UDP-glucuronosyltransferase 1 in Gilbert's syndrome. N Engl J Med. 1995;333:1171-5.

24. Horsfall L, Nazareth I, Pereira SP, Petersen I. Gilbert's syndrome and the risk of death: a population-based cohort study. J Gastroenterol Hepatol. 2013;28:1643-7.

25. Cox AJ, Ng MC, Xu J, Langefeld CD, Koch KL, Dawson PA, Carr JJ, Freedman Bl, Hsu FC, Bowden DW. Association of SNPs in the UGT1A gene cluster with total bilirubin and mortality in the Diabetes Heart Study. Atherosclerosis. 2013;229:155-60. 
26. Temme EH, Zhang J, Schouten EG, Kesteloot H. Serum bilirubin and 10-year mortality risk in a Belgian population. Cancer Causes Control. 2001;12:887-94.

27. Ko WF, Helzlsouer KJ, Comstock GW. Serum albumin, bilirubin, and uric acid and the anatomic site-specific incidence of colon cancer. J Natl Cancer Inst. 1994:86:1874-5.

28. Wagner KH, Wallner M, Molzer C, Gazzin S, Bulmer AC, Tiribelli C, Vitek L. Looking to the horizon: the role of bilirubin in the development and prevention of age-related chronic diseases. Clin Sci. 2015;129:1-25.

29. Najib-Farah. Defensive role of bilirubinemia in pneumococcul infection. Lancet. 1937:229:505-6.

Submit your next manuscript to BioMed Central and we will help you at every step:

- We accept pre-submission inquiries

- Our selector tool helps you to find the most relevant journal

- We provide round the clock customer support

- Convenient online submission

- Thorough peer review

- Inclusion in PubMed and all major indexing services

- Maximum visibility for your research

Submit your manuscript at www.biomedcentral.com/submit
Biomed Central 